\title{
Effects of Design Geometries and Nonlinear Losses on Gain in Silicon Waveguides with Erbium-Doped Regions
}

\author{
Feng Qian, Qi Song, En-Kuang Tien, Salih K. Kalyoncu, Yuewang Huang, and \\ Ozdal Boyraz, Member, IEEE
}

\begin{abstract}
Silicon waveguides integrated with doped dielectric gain media may allow the design of planar light sources with electronic control. In this paper, the effects of design geometries and nonlinear losses on the gain in crystalline silicon waveguides with erbium-doped regions are investigated. We show that by using multitrench geometries, the power confinement can be increased and higher gain-to-nonlinear-loss ratio achieved. Net gain can be improved as much as $0.38 \mathrm{~dB} / \mathrm{cm}$ in multitrench waveguides compared to single-trench waveguides.
\end{abstract}

Index Terms-Erbium, integrated optics, laser amplifiers, waveguides.

\section{INTRODUCTION}

$\mathbf{S}$ ILICON light emitters are the missing links for future silicon-based photonic integration. A main challenge is to find a way to attain high quantum efficiency [1]. Many approaches have been employed to obtain optical gain in silicon, ranging from silicon nanocrystals to erbium-doped silicon, and yet there have been few achievements [2]-[5].

Conventionally, light amplification at telecommunication wavelengths has been achieved using erbium-doped low-index host materials, such as erbium-doped oxide glass (phosphosilicate and soda-lime glasses) and ceramic thin films $\left(\mathrm{Al}_{2} \mathrm{O}_{3}\right.$, $\mathrm{Y}_{2} \mathrm{O}_{3}$ ) [6]-[8]. Well-developed fabrication processes and high erbium solubility in a small volume have attracted great interest in these host materials, and hence planar dielectric waveguides are fabricated to deliver wafer-scale light amplifiers. For instance, $\mathrm{Al}_{2} \mathrm{O}_{3}: \mathrm{Er}^{3+}$ waveguides with 0.1 $\mathrm{dB} / \mathrm{cm}$ background loss have been fabricated with low-cost and reliable fabrication processes, $80 \mathrm{~nm}$ gain bandwidth, and up to $2.3 \mathrm{~dB} / \mathrm{cm}$ amplification performance [9]-[11]. The net gain can be further enhanced to $4.1 \mathrm{~dB} / \mathrm{cm}$ in similar waveguide devices using phosphate glass [12].

Silicon slot waveguides, which are submicrometer slots embedded between waveguides, have been experimentally demonstrated to provide a high optical confinement in the slot region that can accommodate possible optical gain media

Manuscript received June 27, 2010; revised September 2, 2010; accepted October 9, 2010. Date of current version February 24, 2011.

The authors are with the Electrical and Computer Science Department, University of California, Irvine, CA 92697 USA (e-mail: fqian@uci.edu; qsong@uci.edu; ektien@gmail.com; skalyonc@uci.edup; hyw815@gmail.com; oboyraz@uci.edu).

Color versions of one or more of the figures in this paper are available online at http://ieeexplore.ieee.org.

Digital Object Identifier 10.1109/JQE.2010.2088379
[13], [14]. Approaches that confine light in low-index structures have been presented to show achievements in CMOScompatible light-emitting devices. To achieve lasing, the gain provided by the active material in the slot region must be high enough to overcome losses in the whole waveguide. One way of achieving this goal is to use horizontal slot waveguides [15], in which slots are sandwiched between amorphous silicon $(\alpha$ silicon). However, the main advantage of using silicon comes from electronic integration. Thus, the use of crystalline silicon is important for enabling electronic integration.

In this paper, we analyze the structure of crystalline silicon waveguides with multiple erbium-doped $\mathrm{Al}_{2} \mathrm{O}_{3}$ regions sandwiched in between. The advantages of the proposed structures are the following. 1) Light amplification at telecommunication wavelengths is induced by erbium-doped $\mathrm{Al}_{2} \mathrm{O}_{3}$. 2) Electronic tuning capabilities such as switching and modulating are provided by the crystalline silicon platform. The theoretical study presented in this paper evaluates the gain and loss competitions in multitrench crystalline silicon waveguides with erbium-doped $\mathrm{Al}_{2} \mathrm{O}_{3}$. Especially, effects of erbium concentration, power confinement, and nonlinear losses on gain are discussed in detail. Different power distribution profiles in the waveguides and erbium concentrations up to $5 \times 10^{20} \mathrm{~cm}^{-3}$ have been considered. In particular, nonlinear losses that mitigate the optical gain, including excited-state absorption (ESA) and upconversion (UC) in erbium-doped $\mathrm{Al}_{2} \mathrm{O}_{3}$ as well as free-carrier absorption in silicon, have been analyzed. Different design geometries and the associated nonlinear losses have been discussed for their impacts on optical gain. Multitrench geometries are shown to ultimately increase the power confinement in the low-index regions and produce higher gainto-nonlinear-loss ratio. We show that net gain can be improved as much as $0.38 \mathrm{~dB} / \mathrm{cm}$ in multitrench waveguides compared to single-trench waveguides.

The rest of this paper is organized as follows. First, we introduce the theoretical model used to calculate gain and losses along the device using physical parameters determined experimentally. Next, we present the device geometries used in our calculations, and, finally, we conclude with key results and discussions.

\section{Theoretical Model And Analysis}

To evaluate signal amplification by erbium ions, power, signal, and amplified spontaneous emissions (ASE) profiles 
along the waveguide, population inversion, and power confinement need to be analyzed. After determining signal and pump power distributions, signal evolution along the waveguide is performed by separating the signal propagation in silicon and in erbium-doped $\mathrm{Al}_{2} \mathrm{O}_{3}$. The theoretical model presented in this paper includes gain and loss mechanisms in erbiumdoped $\mathrm{Al}_{2} \mathrm{O}_{3}$ regions and silicon regions, as summarized in Fig. 1. Prior to modeling, finite element analysis was performed on each waveguide geometry to obtain normalized power distribution. Then, the analysis of pump, signal, and ASE variation in erbium-doped $\mathrm{Al}_{2} \mathrm{O}_{3}$ was carried out based on four-level rate equations, considering the energy levels presented in Fig. 2 [16]-[18]. In the silicon region, this model emphasizes free-carrier-induced nonlinear losses. After evaluating signal, pump, and ASE power in the waveguide at each propagation step, the total power is redistributed in the geometry according to the mode analysis. Given the small geometrical size and design of the waveguides used in this paper, the mode profile was found to be invariant with respect to the propagation direction. Finally, aggregate signal gains of the proposed structures are calculated from the abovementioned analysis at the end of the waveguide [19], [20]. As silicon's absorption coefficient was found to be $\geq 100 \mathrm{~cm}^{-1}$ at shorter wavelengths, pumping at $1.48 \mu \mathrm{m}$ was observed to be the only feasible scheme considered in this model.

The rate equations following the energy levels presented in Fig. 2 can be expressed as [16]-[18]:

$$
\begin{aligned}
\frac{d N_{1}}{d t}= & -W_{12} N_{1}-R_{12} N_{1}+W_{21} N_{2}+R_{21} N_{2}+\frac{N_{2}}{\tau_{2}} \\
& +C_{2} N_{2}^{2}-C_{37} N_{3}^{2} \\
\frac{d N_{2}}{d t}= & W_{12} N_{1}+R_{12} N_{1}-W_{21} N_{2}-R_{21} N_{2} \\
& -\frac{N_{2}}{\tau_{2}}+\frac{N_{3}}{\tau_{3}}-2 C_{2} N_{2}^{2}-W_{24} N_{2} \\
\frac{d N_{3}}{d t}= & -\frac{N_{3}}{\tau_{3}}+\frac{N_{4}}{\tau_{4}}-C_{37} N_{3}^{2} \\
\frac{d N_{4}}{d t}= & -\frac{N_{4}}{\tau_{4}}+C_{2} N_{2}^{2}+W_{24} N_{24} \\
N_{T}= & N_{1}+N_{2}+N_{3}+N_{4} \\
R_{i j}= & \frac{\sigma_{i j}\left(v_{s}\right)}{h v_{s}} I_{s}+\sum_{j=1}^{m} \frac{\sigma_{i j}\left(v_{j}\right)}{h v_{j}}\left[I_{A S E+}+I_{A S E-}\right] \\
W_{i j}= & \frac{\sigma_{i j}\left(v_{P}\right)}{h v_{P}} I_{P} .
\end{aligned}
$$

Cooperative UC and exited-state absorption were taken into account in erbium-doped $\mathrm{Al}_{2} \mathrm{O}_{3}$ by these rate equations. $N_{T}$ is the total erbium concentration, $N_{1}, N_{2}, N_{3}, N_{4}$ are the $\mathrm{Er}^{3+}$ concentrations in the energy levels ${ }^{4} \mathrm{I}_{15 / 2},{ }^{4} \mathrm{I}_{13 / 2},{ }^{4} \mathrm{I}_{11 / 2}$, and ${ }^{4} I_{9 / 2}$, respectively, as shown in Fig. $2, \tau_{2}, \tau_{3}, \tau_{4}$ are the luminescence lifetimes corresponding to each energy level, respectively, and $C_{2}$ is the cooperative UC coefficient. For high erbium concentrations, second-order UC was found to play a critical role and hence was included in this model $\left(C_{37}\right)$. However, at low erbium concentrations $\left(\sim 3 \times 10^{20} \mathrm{~cm}^{-3}\right)$, if the waveguide is carefully designed and fabricated, the effect

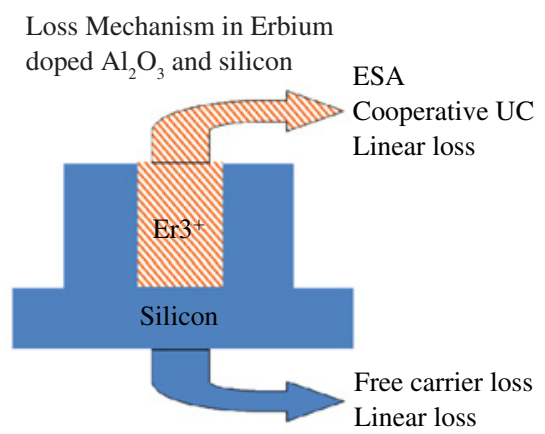

Fig. 1. Gain and loss mechanisms in erbium-doped $\mathrm{AL}_{2} \mathrm{O}_{3}$ and silicon based on the theoretical model in the proposed structures.

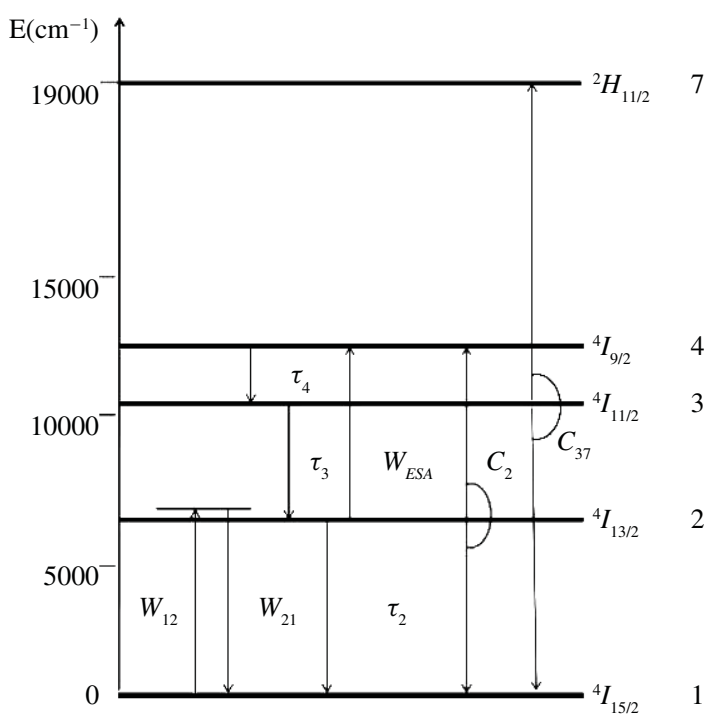

Fig. 2. Energy levels for Er3+ pumped at $1.48 \mu \mathrm{m}$.

of second-order UCs can be negligible and ignored $\left(C_{37}=0\right)$ [17]. $R_{12}, R_{21}, W_{12}$, and $W_{21}$ are the induced signal and pump transition rates, $W_{E S A}$ is the induced ESA rate, $I_{s}, I_{p}$, and $I_{A S E \pm}$ are the signal, pump, and ASE intensities, respectively, and $\sigma_{i j}$ is the emission or absorption cross section. The physical parameters used for numerical analysis are from the experimental data shown in Table I. Steady-state population densities $N_{1}, N_{2}, N_{3}$, and $N_{4}$ at each energy level were calculated by setting the left-hand side of (1)-(4) to zero at each propagation step. The steady-state evolution of the pump, signal, and ASE powers in erbium-doped $\mathrm{Al}_{2} \mathrm{O}_{3}$ are expressed as the following propagation equations [18]:

$$
\begin{aligned}
& \frac{d P_{p}(z)}{d z}=-\gamma_{p}(z) P_{p}(z)-\alpha_{p} P_{p}(z) \\
& \frac{d P_{S}(z)}{d z}=\left[\gamma_{21}\left(z, v_{s}\right)-\gamma_{12}\left(z, v_{S}\right)\right] P_{S}\left(z, v_{s}\right)-\alpha_{S} P_{S}(z) \\
& \frac{d P_{A S E+}\left(z, v_{j}\right)}{d z}=\left[\gamma_{21}\left(z, v_{j}\right)-\gamma_{12}\left(z, v_{j}\right)\right] P_{A S E+}\left(z, v_{j}\right) \\
& +M h v_{j} \Delta v_{j} \gamma_{21}\left(z, v_{j}\right)-\alpha_{S} P_{A S E+}\left(z, v_{j}\right) \\
& \frac{d P_{A S E-}\left(z, v_{j}\right)}{d z}=-\left[\gamma_{21}\left(z, v_{j}\right)-\gamma_{12}\left(z, v_{j}\right)\right] P_{A S E+}\left(z, v_{j}\right) \\
& -M h v_{j} \Delta v_{j} \gamma_{21}\left(z, v_{j}\right)+\alpha_{S} P_{A S E+}\left(z, v_{j}\right) .
\end{aligned}
$$


Here, the solution is obtained for boundary values of

$$
\begin{gathered}
P_{p}(0)=P_{p}, P_{s}(0)=P_{s}, \\
P_{A S E+}\left(0, v_{j}\right)=P_{A S E-}\left(L, v_{j}\right)=0(j=1,2, \ldots, m)
\end{gathered}
$$

where $m$ is the number of frequency slots for ASE. The coefficient $M$ in (10) and (11) represents the number of guided modes at the signal wavelength. The absorption and emission coefficients $\gamma_{12}\left(z, v_{s}\right), \gamma_{21}\left(z, v_{s}\right)$, and $\gamma_{p}(z)$ are given by

$$
\begin{aligned}
\gamma_{12}\left(z, v_{s}\right)= & \iint_{A} \psi_{s}(x, y) \sigma_{12}\left(v_{s}\right) N_{1}(x, y, z) d x d y \\
\gamma_{21}\left(z, v_{s}\right)= & \iint_{A} \psi_{s}(x, y) \sigma_{21}\left(v_{s}\right) N_{2}(x, y, z) d x d y \\
\gamma_{p}(z)= & \iint_{A} \psi_{p}(x, y)\left[\sigma_{12}\left(v_{p}\right) N_{1}(x, y, z)\right. \\
& \left.-\sigma_{21}\left(v_{p}\right) N_{2}(x, y, z)+\sigma_{24}\left(v_{p}\right) N_{2}(x, y, z)\right] d x d y
\end{aligned}
$$

where $\gamma_{p}(x, y)$ and $\gamma_{s}(x, y)$ are normalized pump and signal intensity profiles in the erbium-doped regions, respectively. $\Psi_{s}(x, y)$ and $\Psi_{p}(x, y)$ are the normalized signal and pump intensity profile. $A$ is the cross-sectional area of the erbiumdoped active region, and $\sigma_{12}, \sigma_{21}$, and $\sigma_{24}$ are the absorption and emission cross sections.

With regard to free-carrier absorption in silicon, at each propagation step, $I_{S i}(x, y, z)$ represents the variation of pump intensity at each individual point $\langle x, y, z\rangle$ in silicon, and can be expressed as [21]

$$
\frac{d I_{S i}(x, y, z)}{d z}=-\alpha_{f c a}(z) I_{S i}(x, y, z)-\beta I_{S} i^{2}(x, y, z)
$$

where

$$
\alpha_{f c a}(z)=1.45 \times 10^{-17}\left(\frac{\lambda}{1.55}\right)^{2} N(x, y, z) \quad \mathrm{cm}^{-1} .
$$

$\beta$ represents the two-photon absorption coefficient and $\alpha_{f c a}(z)$ represents the free-carrier absorption expressed in [22]. Also, $N(x, y, z)$ is the free-carrier density at each individual point in silicon and it is deduced from [21, Eq. (6)]

$$
N(x, y, z)=\frac{\tau_{0} \beta I_{S i}^{2}(x, y, z)}{2 h v}+f(i) .
$$

Here, $\tau_{0}$ is the free carrier recombination lifetime. The last term $f(i)$ is the free-carrier absorption induced by current injection in the event of electronic modulation. Since electronic manipulation is not discussed in this paper, freecarrier absorption induced by carrier injection is ignored in the following numerical calculations. At each propagation step, the pump power in silicon affected by free-carrier absorption can be represented as [21]

$$
P_{s i}(z)=\iint_{S i} I_{s i}(x, y, z) d x d y .
$$

Upon determination of the pump profile along the waveguide, the free-carrier losses of the pump can be estimated as

$$
\Delta P_{s i}=P_{s i}(z+1)-P_{s i}(z) .
$$

Numerical calculations by pumping at $1.48 \mu \mathrm{m}$ are summarized as follows.
TABLE I

Physical Parameters Used for Numerical ANalysis of Silicon WaVeguide With ERbiUm-Doped ACtive Regions [16]-[18]

\begin{tabular}{ll}
\hline Parameter & Value \\
\hline Waveguide length & $1 \mathrm{~cm}$ \\
Pump wavelength & $1.48 \mu \mathrm{m}$ \\
Signal power & $1 \mu \mathrm{W}$ \\
Er concentration $\left(N_{E r}\right)$ & $5 \times 10^{19}-5 \times 10^{20} \mathrm{~cm}^{-3}$ \\
Pump absorption cross & $2.7 \times 10^{-21} \mathrm{~cm}^{2}$ \\
$\quad$ section $(1.48 \mu \mathrm{m}) \sigma_{12}$ & \\
Pump emission cross & $0.77 \times 10^{-21} \mathrm{~cm}^{2}$ \\
$\quad$ section $(1.48 \mu \mathrm{m}) \sigma_{21}$ & $5.8 \times 10^{-21} \mathrm{~cm}^{2}$ \\
Signal absorption cross & \\
$\quad$ section $(1.53 \mu \mathrm{m}) \sigma_{12}$ & $6.1 \times 10^{-21} \mathrm{~cm}^{2}$ \\
Signal emission cross & $0.85 \times 10^{-21} \mathrm{~cm}^{2}$ \\
section $(1.53 \mu \mathrm{m}) \sigma_{21}$ & $\left(2.65 \times N_{E r} / 10^{20}+3.38\right)$ \\
ESA cross & $\times 10^{-18} \mathrm{~cm}^{3} / \mathrm{s}$ \\
$\quad$ section $(1.48 \mu \mathrm{m}) \sigma_{24}$ & \\
Cooperative UC $C_{2}$ & $\left(1 \times 10^{-17}-1 \times 10^{-15}\right) \mathrm{cm}^{3} / \mathrm{s}$ \\
& $7.8 \mathrm{~ms}$ \\
Second-order cooperative & $30 \mu \mathrm{s}$ \\
UC $C_{37}$ & $1 \mathrm{~ns}$ \\
${ }^{4} I_{13 / 2}$ state lifetime $\tau_{2}$ & 1.64 \\
${ }^{4} I_{11 / 2}$ state lifetime $\tau_{3}$ & 3.48 \\
${ }^{4} I_{9 / 2}$ state lifetime $\tau_{4}$ & \\
Refractive index of erbium & \\
doped active regions & Refractive index of silicon waveguide
\end{tabular}

1) The normalized pump and signal intensity profiles, i.e., $\Psi_{s}(x, y)$ and $\Psi_{p}(x, y)$, for the fundamental transverseelectric (TE) mode are calculated in erbium-doped $\mathrm{Al}_{2} \mathrm{O}_{3}$ using the finite element method by COMSOL Multiphysics, maintaining the intensity distributions for both signal and ASE. Here, TE polarization is defined as the one in which the electric field vector is parallel to the waveguide slab.

2) At each step in the propagation direction, by solving (1)-(4), $\mathrm{Er}^{3+}$ populations at energy levels $N_{1}-N_{4}$ at each point in the erbium region are calculated.

3) Absorption and emission coefficient $\gamma_{12}\left(z, v_{s}\right)$, $\gamma_{21}\left(z, v_{s}\right)$, and $\gamma_{p}(z)$ are calculated using (12)-(14), through $\Psi_{s}(x, y), \Psi_{p}(x, y), N_{1}$ and $N_{2}$.

4) Numerical integration of propagation equations (8)-(9) is achieved by the Runge-Kutta based iterative procedure to obtain $P_{p}(z)$ and $P_{s}(z)$ at each step.

5) The total pump power is then further attenuated by the nonlinear pump power loss in silicon $\left(\Delta P_{s i}\right)$ at each propagation step as calculated using (19).

\section{Device Geometry}

The device structures of silicon rib waveguides with multiple erbium-doped $\mathrm{Al}_{2} \mathrm{O}_{3}$ regions used are depicted in Fig. 3 . In these structures, the silicon (refractive index $n_{s i}=3.48$ ) rib waveguide acts as a platform to hold rectangular lowindex erbium-doped $\mathrm{Al}_{2} \mathrm{O}_{3}\left(n_{E r-\mathrm{Al}_{2} \mathrm{O}_{3}}=1.64\right)$ regions. In particular, two groups of waveguide geometries (WG1 and WG2 of Fig. 3) are investigated in detail to evaluate the effects of power distribution on nonlinear losses. The waveguide height was fixed at $0.8 \mu \mathrm{m}$ and waveguide widths of $0.6 \mu \mathrm{m}$ and $1 \mu \mathrm{m}$ were selected for the groups WG1 and WG2, respectively. Selection of waveguide width was in accordance with 
the desired single-mode condition in both polarizations, as well as accommodating several low-index erbium-doped active regions [23]. Minimal trench widths and spacings between the trenches were designed as $0.1 \mu \mathrm{m}$ owing to practical fabrication precision, complexity, and cost. As a result, the maximum number of trenches was constrained for different waveguide width designs. The length of the waveguide was selected to be $1 \mathrm{~cm}$ due to limitations of practical fabrication precision, complexity, and cost. For WG1 in Fig. 3(a), two subgroups, i.e., single trench (WG1A) and two trenches (WG1B), configurations were proposed and analyzed. For WG2 in Fig. 3(b), two trenches (WG2A) and four trenches (WG2B) were proposed and analyzed. In the above-mentioned designs, a maximum of two trenches (WG1B) for WG1 were designed with a total active area of $0.2 \mu \mathrm{m} \times 0.7 \mu \mathrm{m}$ and a maximum of four trenches (WG2B) for WG2 were designed with a total active area of $0.4 \mu \mathrm{m} \times 0.7 \mu \mathrm{m}$. For the same silicon waveguide dimensions (width and height), the total erbium-doped active area remained the same for different numbers of active regions (subgroup A or B), showing the effects of trench numbers. By using waveguides with different widths (WG1 and WG2), our goal was to demonstrate how the power distributions and loss mechanisms in the waveguide are affected.

\section{RESUlTS AND Discussion}

Signal amplification in the proposed structures strongly depends on erbium concentration as well as nonlinear loss mechanisms such as cooperative UC, ESA in erbium-doped $\mathrm{Al}_{2} \mathrm{O}_{3}$ regions, and free-carrier absorption in silicon, as illustrated in Fig. 1. Among these loss mechanisms, linear loss of the waveguide is the major reason for amplifier performance degradation and mainly results from the losses at the lateral walls of the waveguide during the etching process. However, since linear loss is not a design parameter, but rather a consequence of the fabrication processes, it will not be discussed in these calculations for device characterization and optimization.

\section{A. Effects of Power Confinement and Population Inversion}

As net gain and nonlinear losses are highly influenced by the optical intensity distribution, the impact of intensity distribution facilitated by different geometries (number of trenches) on each loss mechanism needs to be studied to determine the optical gain. The mode distributions in trenched waveguides are calculated using finite element method provided by COMSOL Multiphysics. From these simulations, the confinement factor and the distribution of normalized power intensity in erbium-doped $\mathrm{Al}_{2} \mathrm{O}_{3}$ regions are extracted for the fundamental TE mode, as illustrated in Fig. 4(a) and (b), respectively. In this structure, TE polarization is characterized as the one in which the electric field vector is perpendicular to the trenches to provide high power confinement in the $\mathrm{Er}^{3+}$ regions. To have a fair comparison, the total volume of the $\mathrm{Er}^{3+}$-doped active regions is set to be equal for the same waveguide group (same waveguide width).

In the analyzed devices, the degree of $\mathrm{Er}^{3+}$ population inversion in the slot regions is plotted along the length of

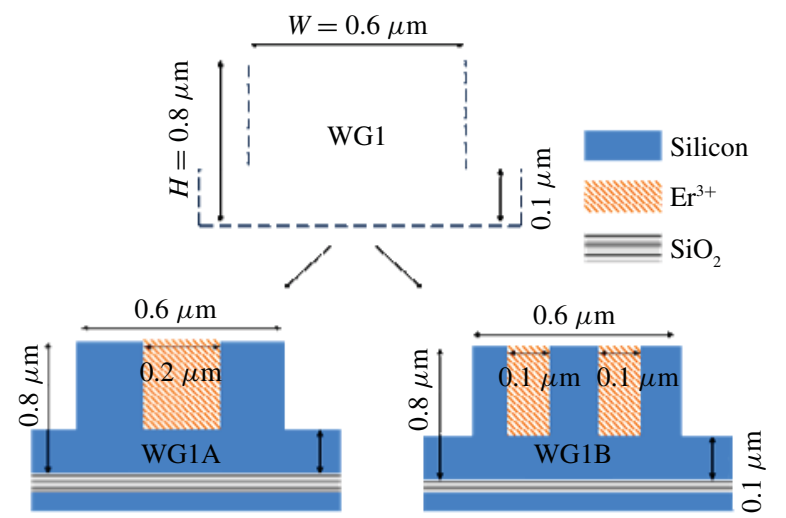

(a)

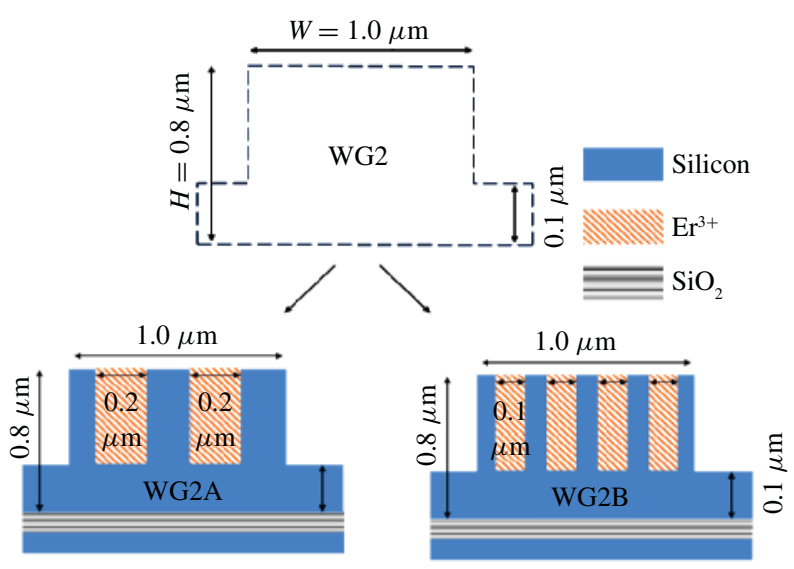

(b)

Fig. 3. (a) Illustrations of WG1 of waveguide of dimensions $0.6 \mu$ m width, $0.8 \mu \mathrm{m}$ height, $0.1 \mu \mathrm{m}$ trench width, WG1A (single trench), and WG1B (two trenches); waveguide length $=1 \mathrm{~cm}$. (b) Illustrations of WG2 of waveguide of dimensions $1 \mu \mathrm{m}$ width, $0.8 \mu \mathrm{m}$ height, $0.1 \mu \mathrm{m}$ trench width, WG2A (two trenches), and WG2B (four trenches); waveguide length $=1 \mathrm{~cm}$.

the waveguide as shown in Fig. 5. The population inversion of $\mathrm{Er}^{3+}$ is integrated over the thickness of the waveguide. The dip in the center of the waveguide is due to ESA. The effect of ESA does not decrease over the length of the waveguide since linear loss in the waveguide is not considered. If linear waveguide loss is considered, the effect of ESA decreases along the length of the waveguide as a result of absorption by $\mathrm{Er}^{3+}$ and waveguide losses, resulting in little or no dip at the end of the waveguide [24].

\section{B. Effects of Second-Order UC and Er ${ }^{3+}$ Concentration}

Second-order UC is known to cause fluorescence at multiple wavelengths, such as $520 \mathrm{~nm}$ green emission due to transitions from the ${ }^{2} \mathrm{H}_{11 / 2}$ state to the ground state, along with fluorescence at 545, 660, and $850 \mathrm{~nm}$ [16], [17], [25], [26]. In particular, second-order UC is the leading detrimental effect at high erbium concentrations of $\geq 1 \times 10^{21} \mathrm{~cm}^{-3}$ [16]. A theoretical estimate of the effects of the second-order UC has been performed by varying the second-order UC coefficient $C_{37}$ based on the reported value [17] in (1)-(4). As the intensity distribution in the waveguide is nonuniform and the $C_{37}$ is intensity-dependent, population inversions are calculated at different geometrical locations [points A-I in 


\begin{tabular}{|l|l|c|}
\hline WG1 group & WG1A & WG1B \\
\hline $\begin{array}{l}\text { Power confinement in } \\
\text { erbium doped } \mathrm{Al}_{2} \mathrm{O}_{3}\end{array}$ & $16.04 \%$ & $27.52 \%$ \\
\hline WG2 group & WG2A & WG2B \\
\hline $\begin{array}{l}\text { Power confinement in } \\
\text { erbium doped } \mathrm{Al}_{2} \mathrm{O}_{3}\end{array}$ & $22.82 \%$ & $33.85 \%$ \\
\hline
\end{tabular}

(a)

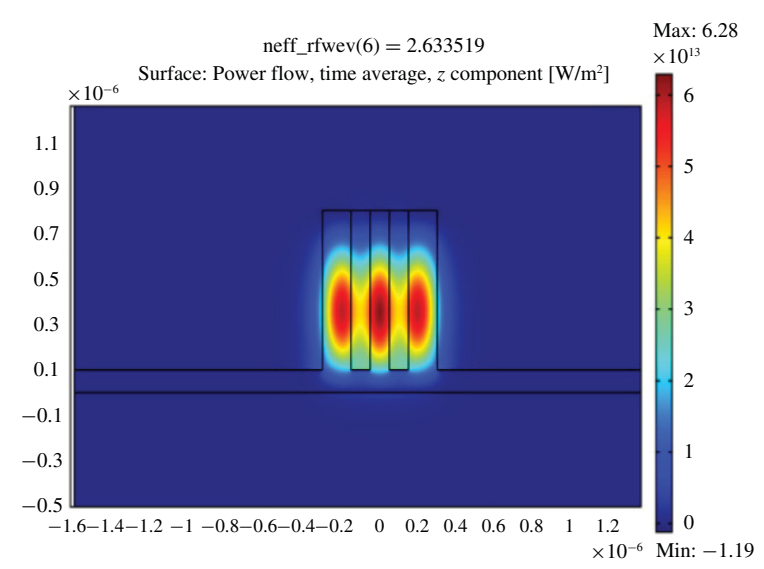

(b)

Fig. 4. (a) Power confinement factors of different proposed geometries. (b) Illustration of normalized power intensity profile $\left(\right.$ per $\mathrm{m}^{2}$ ) of a twotrenched waveguide (WG1B) in WG1 group.

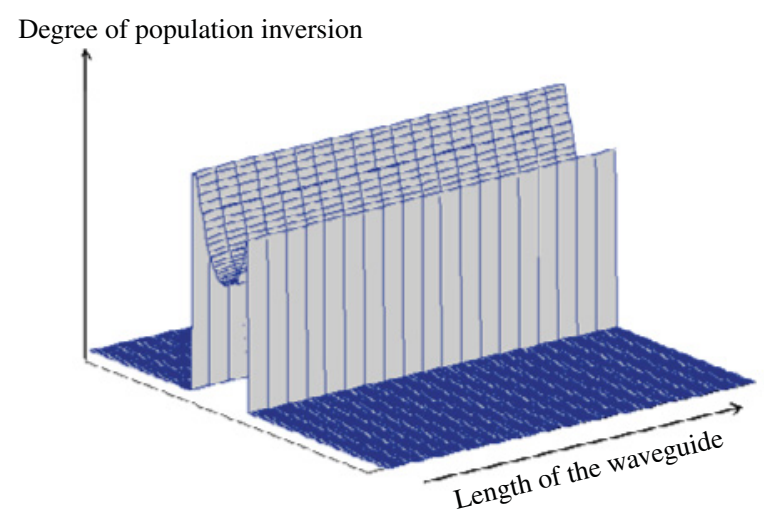

Fig. 5. Degree of inversion along the length of the waveguide. Calculations are made for WG1B, pumped at $100 \mathrm{~mW}$ at $1.48 \mu \mathrm{m}$ (waveguide length $=$ $1 \mathrm{~cm})$.

Fig. 6(a)] in the erbium-doped $\mathrm{Al}_{2} \mathrm{O}_{3}$ region. Here, each point corresponds to different pump intensities, as indicated by the contour lines in Fig. 6(a). By using $C_{37}=8 \times 10^{-16} \mathrm{~cm}^{3} / \mathrm{s}$ [17], we have shown that the population inversions decrease by at least $30 \%$ as depicted in Fig. 6(b). Furthermore, secondorder UC may not lead to an overall net population inversion depending on the intensity distributions in the geometry, as indicated in Fig. 6(b) and in [16], [17], and [26].

Although high erbium solubility in $\mathrm{Al}_{2} \mathrm{O}_{3}$ up to $3 \times 10^{21}$ $\mathrm{Er} / \mathrm{cm}^{3}$ is achievable [26], steady-state erbium population contributing to signal amplifications is greatly reduced by the second-order UC. Thus, high erbium concentration (e.g., $\geq 1 \times$ $10^{21} \mathrm{Er} / \mathrm{cm}^{3}$ ) is not suitable for this [26]. High-order emission peaks are strongly reduced at low erbium concentration of

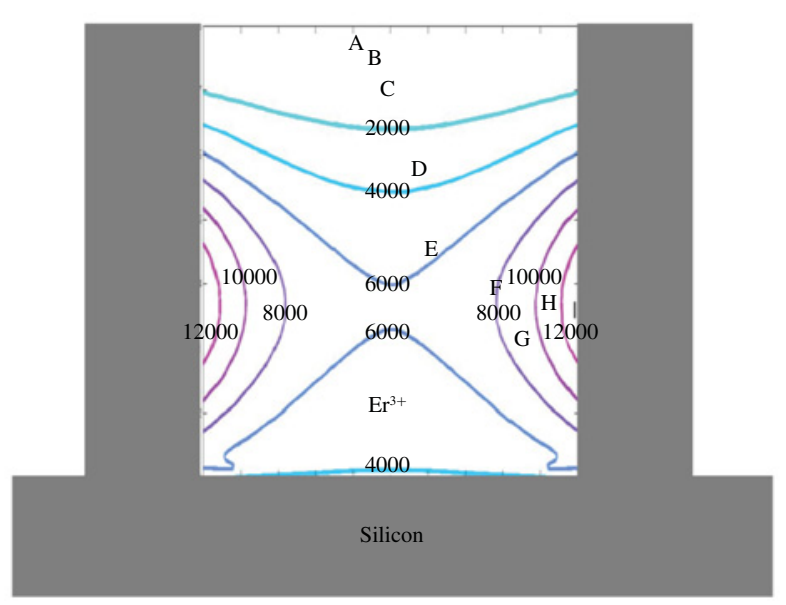

(a)

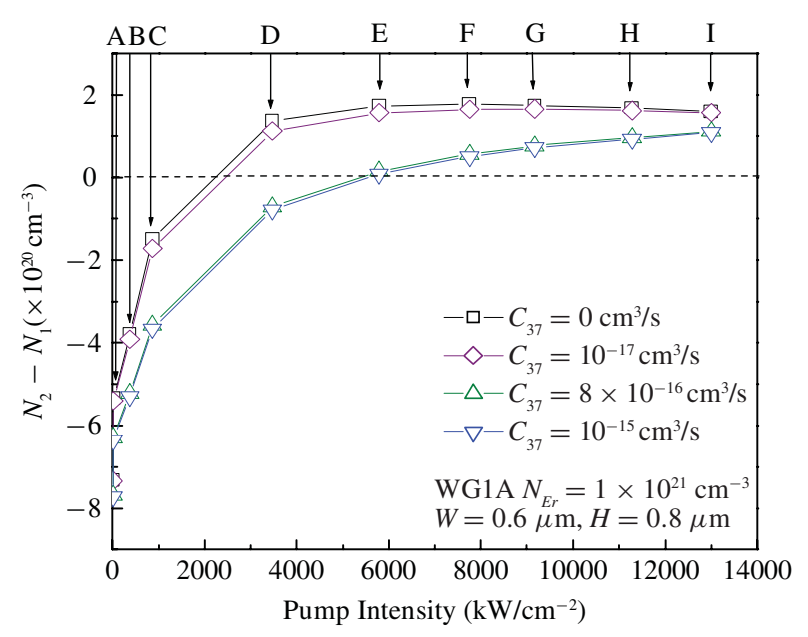

(b)

Fig. 6. (a) Contour plot of pump intensity distribution within erbium-doped $\mathrm{Al}_{2} \mathrm{O}_{3}$. Intensity distributions at exemplary points A-I show the estimated population inversion at locations with different pump intensities. Numbers on the contour lines represent intensity $\left(\mathrm{kw} / \mathrm{cm}^{-3}\right.$ ) (e.g., 2000 represents 2000 $\mathrm{kw} / \mathrm{cm}^{-3}$ ). (b) Population inversion calculated at points A-I with respect to different pump intensities and different values of $\mathrm{C}_{3} 7$.

$\leq 5 \times 10^{20} \mathrm{Er} / \mathrm{cm}^{3}$, thus second-order UC can be neglected if the device is strategically fabricated and engineered [16], [17]. Subsequently, ESA and UC (excluding second-order $\mathrm{UC}$ in this paper) are the detrimental effects that need to be incorporated at lower concentrations. Optimum erbium concentration of $5 \times 10^{20} \mathrm{Er} / \mathrm{cm}^{3}$ is used in the following analysis to provide high optical gain as depicted in Fig. 7, without second-order UC degradation.

\section{ESA and Cooperative UC}

Although there have not been direct measurements of ESA in erbium-doped $\mathrm{Al}_{2} \mathrm{O}_{3}$ with $1.48 \mu \mathrm{m}$ pump wavelength, the ESA process from ${ }^{4} \mathrm{I}_{13 / 2}$ to ${ }^{4} \mathrm{I}_{9 / 2}$ is taken into account based on the experimental results for the gain calculation of erbiumdoped planar waveguides and erbium-doped fiber amplifiers [11], [16], [17], [26], [27]. The ESA process is observed at lower erbium concentrations and explained as a phononassisted process [16]. Thus, the ESA cross section determined from the experimental data is host-material dependent and 


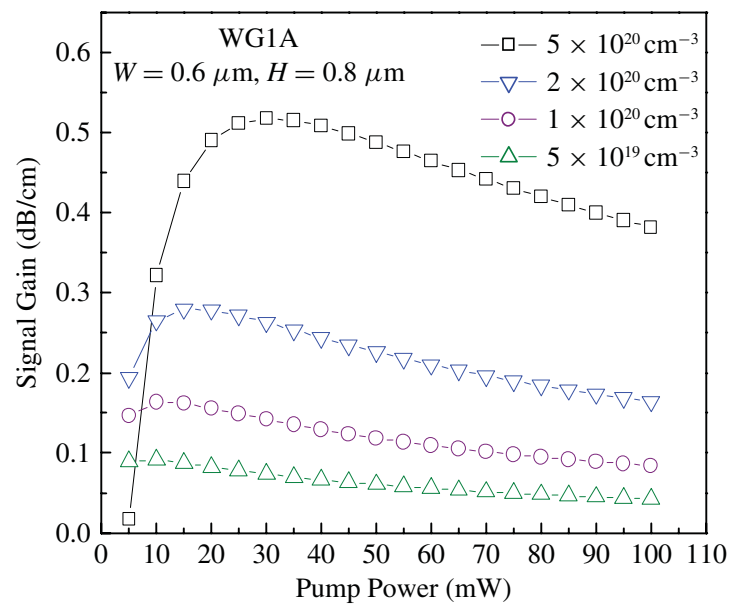

Fig. 7. Signal gains of WG1A versus pump powers of different erbium doping concentrations $\left(5 \times 1020 \mathrm{~cm}^{-3}, 2 \times 1020 \mathrm{~cm}^{-3}, 1 \times 1020 \mathrm{~cm}^{-3}\right.$, $5 \times 1019 \mathrm{~cm}^{-3}$ ), waveguide length $=1 \mathrm{~cm}$.

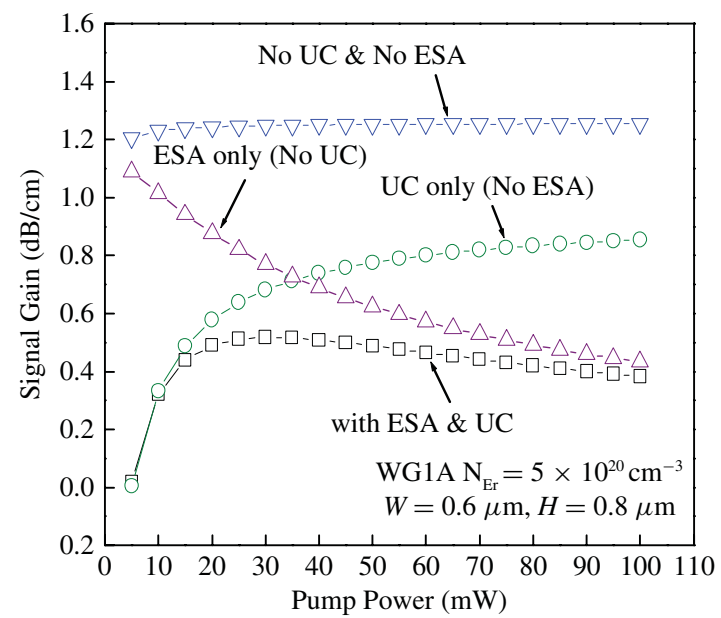

Fig. 8. Signal gains of WG1A with no ESA and UC, no UC, no ESA, and with both ESA and UC (waveguide length $=1 \mathrm{~cm}$ ).

nearly a factor of 10 smaller than the absorption cross section for excitation of the first excited state [16].

In this paper, by using the physical parameters determined experimentally [11], [16], ESA is found to deplete the optical gain at high pump intensities, especially at $1.48 \mu \mathrm{m}$ pump wavelength. As illustrated in Fig. 8, ESA is the strongest effect that reduces the gain at pump powers above $20 \mathrm{~mW}$. Similarly, the cooperative UC process is affected by pump intensity at higher erbium concentration, and gain saturation is introduced at low pump power values, as shown in Fig. 8. The gain profile generated by single-trench (WG1A) and double-trench (WG1B) waveguides are shown in Fig. 9(a). These results indicate that device performance will be limited to low pump operations due to nonlinear losses in erbium-doped regions. Up to $0.38 \mathrm{~dB} / \mathrm{cm}$ more signal gain is provided in the doubletrench configuration when compared with the single-trench design, as shown in Fig. 9(a).

Since linear loss is not considered in this analysis, nonlinear losses in the structures are calculated as

$$
L_{N F}=G_{s a t}-G
$$

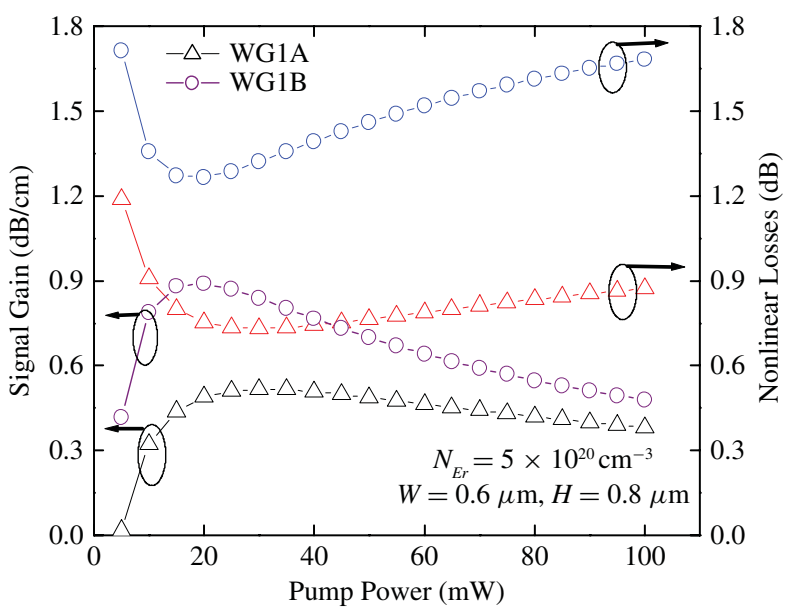

(a)

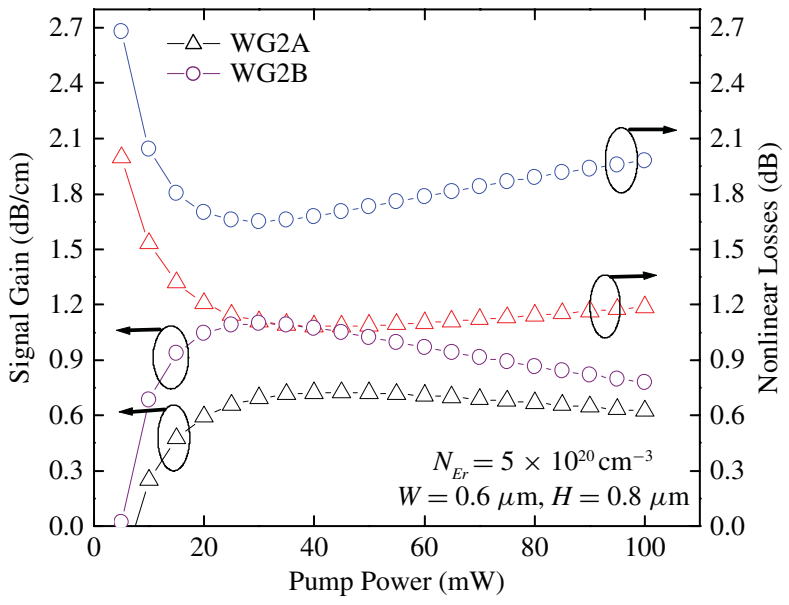

(b)

Fig. 9. (a) Signal gain and nonlinear losses versus pump power for WG1A and WG1B (waveguide length $=1 \mathrm{~cm}$ ). (b) Signal gain and nonlinear losses versus. pump power for $\mathrm{WG} 2 \mathrm{~A}$ and $\mathrm{WG} 2 \mathrm{~B}$ (waveguide length $=1 \mathrm{~cm}$ ).

where $G_{s a t}$ is the saturation gain without linear and nonlinear losses and $G$ is net optical gain considering nonlinear losses.

$G_{\text {sat }}$ is estimated as

$$
G_{\text {sat }}=4.43 \times\left(\sigma_{21} N_{2}-\sigma_{12} N_{1}\right) \Gamma L
$$

where $\sigma_{12}$ and $\sigma_{21}$ are the $\mathrm{Er}^{3+}$ absorption and emission cross sections, $\Gamma$ is the power confinement factor, and $L$ is the waveguide length. As shown in Fig. 9(a), nonlinear losses are increased in double-trench structures. It is mainly caused by the nonuniformity of the power intensity profile in multitrench structures. The improved signal gain of the double-trench waveguide is mainly due to the improved power confinement factor, which improves the saturated gain without linear and nonlinear losses. Larger waveguides can accommodate more erbium-doped regions and have higher power confinement, as seen in Fig. 6(a). The maximum achievable signal gain is improved in the larger waveguides by at least $0.3 \mathrm{~dB} / \mathrm{cm}$, comparing the four-trench configuration (WG2B) in Fig. 9(b) with the two-trench configuration (WG1B) in Fig. 9(a). With no linear loss in the system, as shown in Fig. 9(b), the four-trench design can achieve the maximum gain of $1.1 \mathrm{~dB} / \mathrm{cm}$. 


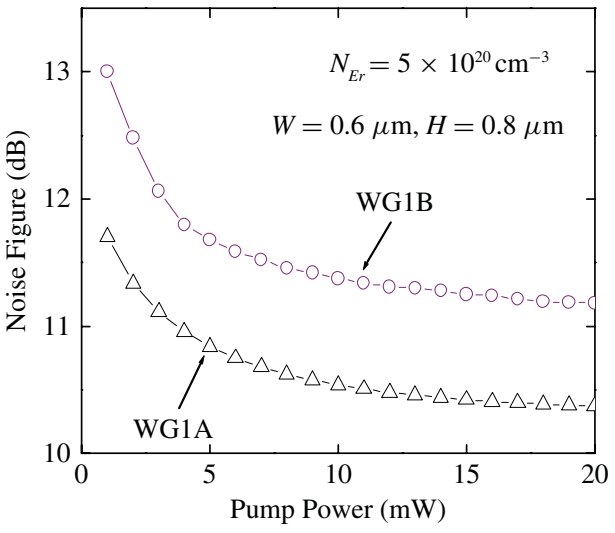

Fig. 10. Noise figure versus input pump power for WG1B and WG1A (waveguide length $=1 \mathrm{~cm}$ ).

Linear loss due to scattering losses on the slot sidewalls is another parameter that should be considered. However, since linear loss is strictly a fabrication-dependent parameter, it is intentionally left out in this analysis. In the presence of linear loss, the effect of ESA decreases along the length of the waveguide as a result of absorption by $\mathrm{Er}^{3+}$ and waveguide losses. In addition, net gain may deplete or vanish depending on the magnitude of the linear loss.

\section{Noise Figure}

The noise figure of the analyzed devices can be easily obtained once the ASE has been spectrally resolved. By considering only signal-spontaneous emission beat noise, the noise figure can be expressed as [28]

$$
N F(d B)=10 \log _{10}\left[\frac{1}{G(z)}+\frac{P_{A S E+}\left(z, v_{s}\right)}{G(z) h v_{s} \delta v}\right] .
$$

$G(z)$ is the signal gain, $v_{s}$ is the signal frequency, and $\delta v$ is the width of the frequency slot used to compute $P_{A S E+}\left(z, v_{s}\right)$. In this paper, the range of ASE spectrum calculated is 1.45$1.65 \mu \mathrm{m}$ and $\delta v=128 \mathrm{GHz}$. The noise figure analysis only applies to the unsaturated gain regime and evaluates the performance of the integrated amplifiers. Due to the nonuniformity of the pump profile, the signal-to-noise ratio is degraded in the multitrench waveguide as shown in Fig. 10. The multitrench waveguide has higher power confinement and population inversion, which leads to more spontaneous decay.

\section{E. Free-Carrier Absorption}

At high pump powers, optical loss induced by free-carrier absorption in silicon cannot be neglected [21]. As the maximum pump power in the proposed waveguide structures are limited by the ESA and UC, we expect to have limited freecarrier losses in the silicon region. For pump power lower than $100 \mathrm{~mW}$ (to minimize ESA and UC effects), the signal gain tends to decrease to $3 \times 10^{-4} \mathrm{~dB} / \mathrm{cm}$, as shown in Fig. 11 . These results indicate that the effect of the free-carrier loss is negligible for pump powers less than $100 \mathrm{~mW}$.

In addition, photon absorption in silicon due to the emissions from $\mathrm{N}_{3}$ and $\mathrm{N}_{4}$ states in the erbium-doped $\mathrm{Al}_{2} \mathrm{O}_{3}$

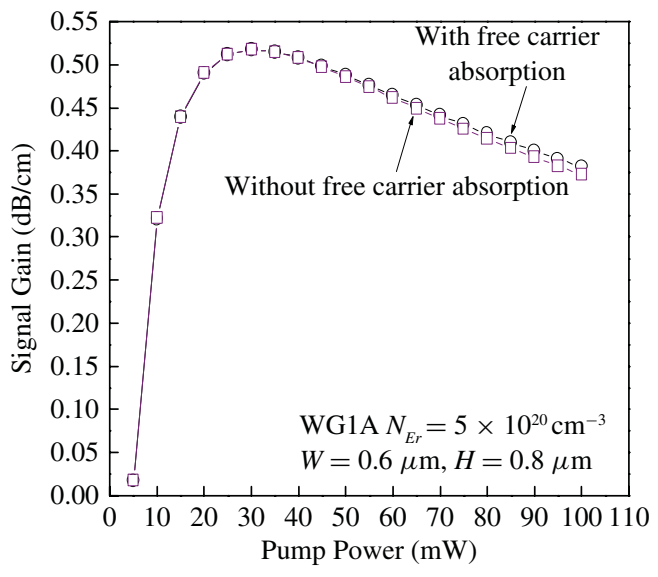

Fig. 11. Signal gain versus pump power for WG1A with and without freecarrier absorption in silicon (waveguide length $=1 \mathrm{~cm}$ ).

regions is also considered. The upper state emissions disappear immediately in the silicon nearby and may become the free carriers, because the absorption coefficient of the $\mathrm{Si}$ layer is large, about $1000 \mathrm{~cm}^{-1}$. Given the erbium ion population in $\mathrm{N}_{3}$ and $\mathrm{N}_{4}$ states (approximately $10^{18} \mathrm{~cm}^{-3}$ ), lifetime of $\mathrm{N}_{3}$ and $\mathrm{N}_{4}$ states (approximately in the order of hundreds of microseconds) and the electron-hole recombination time (approximately in the order of nanoseconds), the generated free-carrier density is estimated to be approximately $10^{10} / \mathrm{cm}^{3}$ and thus it is negligible.

\section{CONClusion}

In conclusion, the effects of design geometries and nonlinear losses on gain in silicon waveguides with erbium-doped $\mathrm{Al}_{2} \mathrm{O}_{3}$ regions were studied. The performance of silicon waveguides with erbium-doped $\mathrm{Al}_{2} \mathrm{O}_{3}$ regions was limited by the UC and ESA. Second-order UC was found to be one of the detrimental effects that prevent signal gain at high erbium concentrations. Distributing erbium ions over multitrench areas improves the gain-to-nonlinear-loss ratio. Thus, $0.38 \mathrm{~dB} / \mathrm{cm}$ higher signal amplification was generated when compared to a single-trench configuration. Linear losses of the analyzed devices are mainly due to the fabrication processes. However, linear losses cannot be ignored in final designs, and thus the fabrication processes need to be improved to provide ultimate high signal amplification.

\section{REFERENCES}

[1] N. Daldosso and L. Pavesi, "Nanosilicon photonics," Laser \& Photon. Rev., vol. 3, no. 6, pp. 508-534, Nov. 2009.

[2] L. Dal Negro, L. Pavesi, G. Pucker, G. Franzo, and F. Priolo, "Optical gain in silicon nanocrystals," Opt. Mater, vol. 17, nos. 1-2, pp. 41-44, Jun.-Jul. 2001.

[3] H. S. Han, S. Y. Seo, and J. H. Shin, "Optical gain at $1.54 \mu \mathrm{m}$ in erbiumdoped silicon nanocluster sensitized waveguide," Appl. Phys. Lett., vol. 79, no. 27, pp. 4568-4570, 2001.

[4] M. Lipson, "Guiding, modulating, and emitting light on siliconchallenges and opportunities," J. Lightw. Technol., vol. 23, no. 12, pp. 4222-4238, Dec. 2005.

[5] X. C. Sun, J. F. Liu, L. C. Kimerling, and J. Michel, "Toward a germanium laser for integrated silicon photonics," IEEE J. Sel. Topics Quantum Electron., vol. 16, no. 1, pp. 124-131, Jan.-Feb. 2010. 
[6] M. Dejneka and B. Samson, "Rare-earth-doped fibers for telecommunications applications," MRS Bull., vol. 24, no. 9, pp. 39-45, Sep. 1999.

[7] C. E. Chryssou, F. Di Pasquale, and C. W. Pitt, " $\mathrm{Er}^{3+}$-doped channel optical waveguide amplifiers for WDM systems: A comparison of tellurite, alumina and Al/P silicate materials," IEEE J. Sel. Topics Quantum Electron., vol. 6, no. 1, pp. 114-121, Jan.-Feb. 2000.

[8] A. J. Kenyon, "Recent developments in rare-earth doped materials for optoelectronics," Progress Quantum Electron., vol. 26, nos. 4-5, pp. 225-284, 2002.

[9] K. Worhoff, J. D. B. Bradley, F. Ay, D. Geskus, T. P. Blauwendraat, and M. Pollnau, "Reliable low-cost fabrication of low-loss $\mathrm{Al}_{2} \mathrm{O}_{3}: \mathrm{Er}^{3+}$ waveguides with 5.4-dB optical gain," IEEE J. Quantum Electron., vol. 45, no. 5, pp. 454-461, May 2009.

[10] J. D. B. Bradley, L. Agazzi, D. Geskus, F. Ay, K. Worhoff, and M. Pollnau, "Gain bandwidth of $80 \mathrm{~nm}$ and $2 \mathrm{~dB} / \mathrm{cm}$ peak gain in $\mathrm{Al}_{2} \mathrm{O}_{3}: \mathrm{Er}^{3+}$ optical amplifiers on silicon," J. Opt. Soc. Am. B, vol. 27, no. 2, pp. 187-196, 2010.

[11] G. N. van den Hoven, R. J. I. M. Koper, A. Polman, C. van Dam, J. W. M. van Uffelen, and M. K. Smit, "Net optical gain at $1.53 \mu \mathrm{m}$ in Er-doped $\mathrm{Al}_{2} \mathrm{O}_{3}$ waveguides on silicon," Appl. Phys. Lett., vol. 68, no. 14, pp. 1886-1888, Apr. 1996.

[12] Y. C. Yan, A. J. Faber, H. de Waal, P. G. Kik, and A. Polman, "Erbiumdoped phosphate glass waveguide on silicon with $4.1 \mathrm{~dB} / \mathrm{cm}$ gain at $1.535 \mu \mathrm{m}, "$ Appl. Phys. Lett., vol. 71, no. 20, pp. 2922-2924, Nov. 1997.

[13] Q. Xu, V. R. Almeida, and M. Lipson, "Experimental demonstration of guiding and confining light in nanometer-size low-refractive-index material," Opt. Lett., vol. 29, no. 14, pp. 1626-1628, Jul. 2004.

[14] V. R. Almeida, Q. F. Xu, C. A. Barrios, and M. Lipson, "Guiding and confining light in void nanostructure," Opt. Lett., vol. 29, no. 11, pp. 1209-1211, Jun. 2004.

[15] R. Sun, P. Dong, N. N. Feng, C. Y. Hong, J. Michel, M. Lipson, and L. Kimerling, "Horizontal single and multiple slot waveguides: Optical transmission at $\lambda=1550 \mathrm{~nm}, "$ Opt. Exp., vol. 15, no. 26, pp. 1796717972, Dec. 2007.

[16] G. N. van den Hoven, E. Snoeks, A. Polman, C. van Dam, J. W. M van Uffelen, and M. K. Smit, "Upconversion in Er-implanted $\mathrm{Al}_{2} \mathrm{O}_{3}$ waveguides," J. Appl. Phys., vol. 79, no. 3, pp. 1258-1266, 1996.

[17] P. G. Kik and A. Polman, "Cooperative upconversion as the gain-limiting factor in Er doped miniature $\mathrm{Al}_{2} \mathrm{O}_{3}$ optical waveguide amplifiers," $J$. Appl. Phys., vol. 93, no. 9, pp. 5008-5012, May 2003.

[18] S. F. Li, C. L. Song, Q. J. Xiong, and B. Ran, "A numerical analysis of gain characteristics of Er-doped $\mathrm{Al}_{2} \mathrm{O}_{3}$ waveguide amplifiers," Opt. Quantum Electron., vol. 34, no. 9, pp. 859-866, 2002.

[19] V. Donzella, S. Faralli, V. Toccafondo, and F. Di Pasquale, "Effect of Si$\mathrm{nc}$ to $\mathrm{Er}^{3+}$ coupling ratio in EDWAs longitudinally pumped by visible broad-area lasers," J. Lightw. Technol., vol. 27, no. 16, pp. 3342-3350, Aug. 2009.

[20] V. Toccafondo, S. Faralli, and F. Di Pasquale, "Evanescent multimode longitudinal pumping scheme for Si-nanocluster sensitized $\mathrm{Er}^{3+}$-doped waveguide amplifiers," J. Lightw. Technol., vol. 26, no. 21, pp. 35843591, Nov. 2008

[21] T. K. Liang and H. K. Tsang, "Role of free carriers from two-photon absorption in Raman amplification in silicon-on-insulator waveguides," Appl. Phys. Lett., vol. 84, no. 15, pp. 2745-2747, Apr. 2004.

[22] E. K. Tien, F. Qian, N. S. Yuksek, and O. Boyraz, "Influence of nonlinear loss competition on pulse compression and nonlinear optics in silicon," Appl. Phys. Lett., vol. 91, no. 20, pp. 201115-1-201115-3, Nov. 2007.

[23] S. H. Yang, M. L. Cooper, P. R. Bandaru, and S. Mookherjea, "Giant birefringence in multi-slotted silicon nanophotonic waveguides," Opt. Exp., vol. 16, no. 11, pp. 8306-8316, May 2008.

[24] A. Polman and F. C. J. M. van Veggel, "Broadband sensitizers for erbium-doped planar optical amplifiers: Review," J. Opt. Soc. Am. B, vol. 21, no. 5, pp. 871-892, 2004.

[25] C. P. Michael, H. B. Yuen, V. A. Sabnis, T. J. Johnson, R. Sewell, R. Smith, A. Jamora, A. Clark, S. Semans, P. B. Atanackovic, and O. Painter, "Growth, processing, and optical properties of epitaxial $\mathrm{Er}_{2} \mathrm{O}_{3}$ on silicon," Opt. Exp., vol. 16, no. 24, pp. 19649-19666, Nov. 2008.

[26] P. G. Kik and A. Polman, "Erbium doped optical-waveguide amplifiers on silicon," MRS Bull., vol. 23, no. 4, pp. 48-54, Apr. 1998.
[27] P. Blixt, J. Nilsson, J. Babonas, and B. Jaskorzynska, "Excitedstate absorption at $1.5 \mu \mathrm{m}$ in $\mathrm{Er}^{3+}$-doped fiber amplifiers," in Proc. Opt. Amplifiers Appl., vol. 17. Sante Fe, NM, Jun. 1992, no. WE2, pp. $1-3$.

[28] F. Dipasquale and M. Federighi, "Modeling of uniform and pair-induced up-conversion mechanisms in high-concentration erbium-doped silica wave-guides," J. Lightw. Technol., vol. 13, no. 9, pp. 1858-1864, Sep. 1995.

Feng Qian is currently working toward the Ph.D. degree in electrical engineering at the Advanced Photonics Devices and Systems Laboratory, University of California, Irvine.

Her current research interests include integrated optics, silicon photonics, silicon based lasers, and waveguide technology.

Qi Song is currently working toward the Ph.D. degree in electrical engineering at the Advanced Photonics Devices and Systems Laboratory, University of California, Irvine.

His current research interests include integrated optics, microwave photonics, and optical communication systems.

En-Kuang Tien is currently working toward the Ph.D. degree in electrical engineering at the Advanced Photonics Devices and Systems Laboratory, University of California, Irvine.

His current research interests include silicon photonics and nonlinear optics in silicon.

Salih K. Kalyoncu is currently working toward the Ph.D. degree in electrical engineering at the Advanced Photonics Devices and Systems Laboratory, University of California, Irvine.

His current research interests include silicon photonics and nonlinear optics in silicon.

Yuewang Huang is currently working toward the Ph.D. degree in electrical engineering at the Advanced Photonics Devices and Systems Laboratory, University of California, Irvine.

His current research interests include silicon photonics and nonlinear optics in silicon.

Ozdal Boyraz (M'96) received the Ph.D. degree in electrical engineering from the University of Michigan, Ann Arbor, in 2001.

$\mathrm{He}$ joined the University of California, Los Angeles, as a Research Scientist to perform research on silicon based photonic devices and microwave photonics, in 2003, after two years of industry experience at Xtera Communications, Allen, TX, as an Optical Communications System Designer. Since October 2005, he has been working as an Assistant Professor at the Electrical Engineering and Computer Science Department, University of California, Irvine. His current research interests include silicon based photonic devices, nonlinear optical devices, optical communication systems, and optical signal processing.

Dr. Boyraz is a recipient of the Defense Advanced Research Projects Agency Young Faculty Award in 2010. 